\section{Diferenciais de mortalidade jovem no Brasil: a importância dos fatores socioeconômicos dos domicílios e das condições de vida nos municípios e estados brasileiros}

\section{Differences in mortality in Brazilian youth:}

the importance of household socioeconomic factors and living conditions in Brazilian municipalities and states

Diferenciales de mortalidad joven en Brasil: la importancia de los factores socioeconómicos de los domicilios y condiciones de vida en los municipios y estados brasileños

\section{Resumo}

Neste artigo, analisou-se a relação entre o nível socioeconômico dos domicílios e das condições sociais locais com o risco de morte de jovens brasileiros. Foram utilizados os dados de óbitos ocorridos nos últimos 12 meses coletados pelo Censo Demográfico de 2010 para realizar a análise em nível local. A análise dos dados foi feita com base em modelos hierárquicos que possibilitaram identificar a contribuição das condições sociais dos domicílios, municípios e estado de residência na mortalidade de jovens no Brasil. Os resultados indicam que as diferenças nos níveis de status socioeconômico entre os domicílios são responsáveis por mais de $90 \%$ da variabilidade no risco de relato de óbito jovem pelo domicílio. Além disso, os resultados mostram que residir em uma localidade com baixo nível socioeconômico aumenta as chances de ocorrência de relato de óbito de jovens pelos domicílios de qualquer nível social no período.

Adulto Jovem; Condições Sociais; Mortalidade
Fabiano Neves Alves Pereira 1,2 Bernardo Lanza Queiroz 2

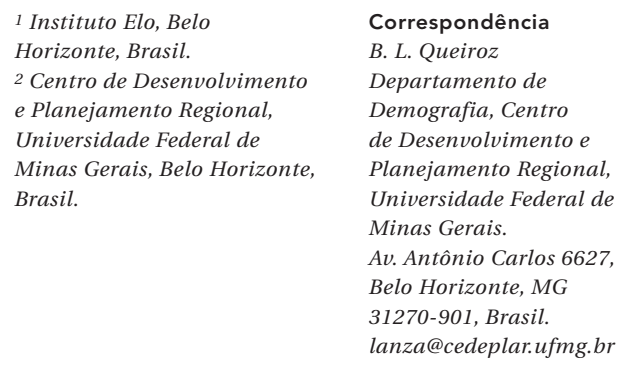

1 Instituto Elo, Belo

Horizonte, Brasil.

2 Centro de Desenvolvimento

e Planejamento Regional

Universidade Federal de

Minas Gerais, Belo Horizonte

Brasil. 


\section{Introdução}

A mortalidade de jovens adultos é um problema social, econômico e de saúde pública 1,2. A mortalidade por causas externas é a principal causa de morte para os jovens de 15 a 29 anos no Brasil 1,2. Uma análise da contribuição das causas externas mostra que a eliminação dessa componente levaria a um aumento de, pelo menos, três anos na esperança de vida ao nascer dos homens 3 . Além disso, elas representam 38\% dos anos de vida perdidos no Brasil no início do século XXI e, em relação ao grupo de jovens adultos, cerca de $70 \%$ do total de anos de vida perdidos 2,3,4. Não apenas as vidas perdidas são um problema, mas o elevado número de óbitos para os jovens de até 29 anos tem um grande peso para as famílias e a sociedade 5,6.

Os fatores associados à mortalidade dos jovens podem ser agrupados em atributos pessoais e contextuais. Em geral, os estudos sobre violência e mortalidade desse grupo etário focam nas condições de vida dos seus respectivos locais de moradia que podem estar associadas a um risco mais elevado de mortalidade do que nas condições domiciliares e do próprio indivíduo 7,8,9,10. Tais estudos são baseados em dados agregados que dimensionam a existência de diferenciais de mortalidade pelas características dos locais de moradia como renda média e condições de infraestrutura 11,12,13,14,15. Há evidências de uma forte correlação negativa entre as taxas de homicídio de jovens e os indicadores socioeconômicos dos distritos, dentre eles o nível de renda domiciliar, indicando desvantagem para os locais com piores condições sociais 16 .

Compreender a magnitude da mortalidade de jovens adultos, a sua distribuição no espaço e a sua relação com características sociais e econômicas em diferentes níveis (família, domicílio e localidade) é de grande importância para aprofundar a discussão sobre essa temática. A identificação das características domiciliares e regionais com maior probabilidade de ocorrência de óbitos de jovens adultos pode ajudar no desenvolvimento de políticas e ações direcionadas a tratar esse problema social, econômico e de saúde. Diante disso, este artigo contribui para a literatura ao testar a relação entre condições de vida e mortalidade para a população jovem tendo como referência as condições socioeconômicas do domicílio do indivíduo e de seu contexto geográfico de residência 17,18,19,20,21,22. Este estudo propõe uma abordagem complementar aos trabalhos tradicionais 1,4 ao conjugar hierarquicamente três esferas das condições de vida nas quais os jovens, considerando homens e mulheres entre 15 e 29 anos de idade, estão inseridos, para analisar os fatores associados à existência de diferenciais de mortalidade, sendo elas a esfera intradomiciliar, municipal e estadual.

\section{Materiais e métodos}

\section{Base de dados e variáveis utilizadas no estudo}

Para investigar os fatores domiciliares e locais associados à mortalidade de jovens no Brasil, usaram-se os dados do Censo Demográfico de 2010. Esse censo incorporou, no seu questionário, quesitos relativos à ocorrência de óbitos nos domicílios no ano anterior ao levantamento. No caso da ocorrência do óbito, foi investigado o sexo e a idade da pessoa que foi a óbito 23,24 . Assim, o Censo Demográfico é utilizado para identificar domicílios que observaram a ocorrência de óbito de jovem nos últimos 12 meses e para a construção de variáveis sobre a situação socioeconômica dos domicílios.

A principal variável explicativa no âmbito dos domicílios é o indicador sintético de status socioeconômico domiciliar. Como não é possível conhecer a coscorendição do domicílio antes da ocorrência do óbito de um de seus membros e se esse evento causou grandes transformações na situação socioeconômica do domicílio, assumiuse que ela não se alterou no intervalo de tempo de um ano. Fundamentado na teoria da associação entre status socioeconômico e mortalidade para construir o indicador, foram utilizadas as seguintes variáveis: nível de instrução do responsável pelo domicílio e renda domiciliar per capita 25,26,27,28. Para calcular o índice, foi necessário reorganizar as informações de educação e renda para que pudessem ser usadas diretamente no indicador sintético proposto.

O Indicador de Escolaridade do Chefe do Domicílio (IECD) foi construído com as informações de escolaridade do censo de 2010. As informações série e graus completos foram reorganizados em níveis de instrução, e cada nível de instrução recebeu uma ponderação específica para ser incluído no indicador final. Os domicílios cujos chefes não tinham educação formal ou Fundamental incompleto, nível baixo de instrução, receberam escore 0,25; aqueles com Fundamental completo e Médio incompleto tiveram escore 0,5; Médio completo e Superior incompleto receberam o escore 0,75 ; e os com Superior completo ficaram com o escore 1 .

A mesma lógica foi utilizada para a construção do Indicador de Renda do Domicílio (IRD), no qual foi utilizada a renda per capita do domicílio obtida do Censo Demográfico. Nesse caso 
para domicílios com renda per capita igual a 0 o escore recebido foi 0 ; entre $\mathrm{R} \$ 0,01$ e $\mathrm{R} \$ 65,00$, o escore foi 0,1; entre R\$ 65,01 e R\$120,00, o escore foi 0,2 ; entre $R \$ 120,01$ e $R \$ 240,00$, o escore foi 0,3; entre $\mathrm{R} \$ 240,01$ e R\$ 400,00, o escore foi 0,4; entre $\mathrm{R} \$ 400,01$ e $\mathrm{R} \$ 675,00$, entre $\mathrm{R} \$ 675,01$ e $\mathrm{R} \$$ $1.350,00$, o escore foi 0,6 ; entre $\mathrm{R} \$ 1.350,01$ e $\mathrm{R} \$$ $3.375,00$, o escore foi 0,8 ; e acima de $\mathrm{R} \$ 3.375,00$, o escore foi 1.

Definidos os escores, as duas variáveis foram integradas para compor o indicador sintético, SSED (Status Socioeconômico do Domicílio), seguindo a Equação 1:

$\operatorname{SSED}_{j}=\frac{\left[\left(p_{1 *} \mid \operatorname{IECD}_{j}\right)+\left(\mathrm{p}_{2}{ }^{*} \mid R D_{j}\right)\right]}{p_{1}+p_{2}}$

Onde: SSED: Status Socioeconômico do Do-

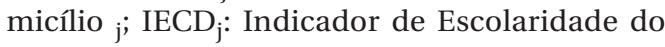
Chefe do Domicílio ${ }_{j}$; IRD ${ }_{j}$ : Indicador de Renda do Domicílio ${ }_{\mathrm{j}} \mathrm{e} ; \mathrm{p}_{1} \mathrm{e}_{2}$ : pesos dos indicadores de escolaridade e renda.

O SSED foi obtido por média ponderada entre os escores de escolaridade (IECD) e renda do domicílio (IRD). Para a definição do peso de cada um dos indicadores parciais de status socioeconômico, utilizou-se como parâmetro a correlação de Pearson entre cada um dos indicadores (escolaridade e renda) e a mortalidade jovem com base nos dados do Censo Demográfico de 2010. O status socioeconômico de escolaridade do domicílio apresentou um nível de correlação com a mortalidade jovem da ordem de $-0,18$. Já o status socioeconômico de renda do domicílio apresentou um nível de correlação com a mortalidade da ordem de $-0,10$. Ao todo, como o grau de correlação de ambas as variáveis com a mortalidade jovem foi de $-0,28$, na equação, a escolaridade recebeu peso proporcional de 0,64 $\left(p_{1}\right)$ e, por sua vez, a renda recebeu peso $0,36\left(p_{2}\right)$. Com base nesses resultados, os domicílios foram classificados segundo status socioeconômico como baixo - escore de 0 a 0,375 ; médio - escore de 0,376 e 0,70; e alto - escore acima de 0,71.

Foram incluídas como variáveis no modelo informações das bases de dados do Índice de Desenvolvimento Humano Municipal (IDH-M) disponibilizada pelo Programa das Nações Unidas para o Desenvolvimento (PNUD). Os dados foram usados como variáveis explicativas no nível dos municípios. O IDH-M consiste numa adaptação do IDH clássico para cada município brasileiro. Juntamente com essa variável que compõe a medida direta das condições de vida nos municípios, foi incorporada ao estudo, como controle, a variável porte populacional dos municípios (com transformação logarítmica). No âmbito das Unidades da Federação foi usada como variável explicativa o IDH.
A Tabela 1 apresenta as variáveis que foram utilizadas no trabalho, a descrição de cada uma delas, a fonte de informação e os códigos de classificação de cada uma delas. Ainda no nível do domicílio, foram utilizadas como controle as variáveis sexo e raça do responsável pelo domicílio.

\section{Modelo analítico}

A hipótese principal supõe que viver em domicílios com baixo nível socioeconômico e/ou em municípios e Unidades da Federação com piores condições de vida aumenta o risco de relato de mortalidade jovem e, consequentemente, contribui para a ampliação dos diferenciais de mortalidade jovem encontrada no Brasil. Dessa forma, usou-se um modelo hierárquico de três níveis em que cada um deles é representado por um submodelo que expressa a relação entre as variáveis dentro de cada nível e também entre os outros níveis 29 . No caso deste trabalho, existem 3.422.261 domicílios representando o primeiro nível e agrupados segundo 5.665 municípios alocados no segundo nível, por sua vez, agrupados segundo as 27 Unidades da Federação do Brasil no terceiro nível.

A metodologia de modelos hierárquicos é uma extensão dos modelos de regressão, uma vez que assume que a variável resposta é definida pela ação de variáveis explicativas, medidas em níveis distintos e, em qualquer um dos níveis, existe variação residual, ou seja, variação que não pode ser explicada pelas variáveis medidas. A incorporação da estrutura hierárquica dos dados na análise se justifica por possibilitar a mensuração de efeitos em diferentes âmbitos sobre o relato de mortalidade jovem pelo domicílio. Portanto, espera-se que esse evento seja influenciado tanto por características específicas dos domicílios como por elementos contextuais dos municípios e Unidade da Federação 29,30.

Neste estudo, foi utilizado um modelo hierárquico porque se supôs que há uma estrutura de dependência entre os domicílios que residem no mesmo município e entre os municípios de um mesmo estado. Dessa forma, nos níveis hierárquicos superiores, municípios e estados, existem variáveis contextuais que podem estar relacionadas com a ocorrência do óbito de um jovem.

Pela estimação do primeiro modelo, é possível estimar a variabilidade relacionada com cada um dos níveis, vale dizer, particionar a variância verificada entre cada um dos três níveis. Mediante isso, o procedimento para identificar também o papel de cada variável em cada um dos níveis do modelo hierárquico consiste em incluir nos modelos as variáveis intrínsecas aos níveis 29,30 . 
Tabela 1

Descrição das variáveis selecionadas

\begin{tabular}{|c|c|c|c|c|}
\hline $\begin{array}{l}\text { Unidade } \\
\text { analítica }\end{array}$ & Variável & Fonte & $\begin{array}{l}\text { Classificação no } \\
\text { modelo }\end{array}$ & Código de categorias \\
\hline \multirow[t]{5}{*}{ Domicílio } & $\begin{array}{l}\text { Ocorreu o óbito de um jovem com } \\
\text { idade entre 15-29 anos no domicílio }\end{array}$ & $\begin{array}{l}\text { Amostra - Censo Demográfico } \\
2010\end{array}$ & Dependente & $\begin{array}{l}0-\text { não } \\
1-\operatorname{sim}\end{array}$ \\
\hline & SSED & $\begin{array}{c}\text { Amostra - Censo Demográfico } \\
2010\end{array}$ & Independente & $\begin{array}{c}0 \text { a } 1 \text { (0 representa a pior condição } \\
\text { e } 1 \text { a melhor condição) }\end{array}$ \\
\hline & $\begin{array}{l}\text { Sexo do responsável } \\
\text { pelo domicílio }\end{array}$ & $\begin{array}{l}\text { Amostra - Censo Demográfico } \\
2010\end{array}$ & Independente & $\begin{array}{l}0 \text { - mulher } \\
1 \text { - homem }\end{array}$ \\
\hline & $\begin{array}{c}\text { Cor/Raça do responsável } \\
\text { pelo domicílio }\end{array}$ & $\begin{array}{c}\text { Amostra - Censo Demográfico } \\
2010\end{array}$ & Independente & $\begin{array}{c}1 \text { - não negro (branco, amarelo, } \\
\text { indígena) }\end{array}$ \\
\hline & & & & 0 - negro (pardo e preto) \\
\hline \multirow[t]{2}{*}{ Município } & IDH-M & PNUD (2013) & Independente & $\begin{array}{c}0 \text { a } 1 \text { (0 representa a pior condição } \\
\text { e } 1 \text { a melhor condição) }\end{array}$ \\
\hline & Porte populacional & Censo Demográfico 2010 & Independente & Logaritmo (população residente) \\
\hline \multirow[t]{2}{*}{ UF } & $\mathrm{IDH}$ & PNUD (2013) & Independente & $\begin{array}{c}0 \text { a } 1 \text { (0 representa a pior condição } \\
\text { e } 1 \text { a melhor condição) }\end{array}$ \\
\hline & Índice Gini & PNUD (2013) & Independente & $\begin{array}{l}0 \text { a } 1 \text { (0 representa a melhor } \\
\text { condição } 1 \text { a pior condição) }\end{array}$ \\
\hline
\end{tabular}

IDH: Índice de Desenvolvimento Humano; IDH-M: Índice de Desenvolvimento Humano Municipal; PNUD: Programa das Nações Unidas para o

Desenvolvimento; SSED: Status Socioeconômico do Domicílio; UF: Unidade da Federação.

Na análise do diferencial de mortalidade, a utilização do modelo hierárquico é devida ao reconhecimento que existe, variabilidade relacionada à localidade (municípios e estados), mesmo após o controle pelas características dos domicílios. Dessa maneira, domicílios semelhantes de municípios e/ou estados distintos podem ter probabilidades distintas de observar um óbito de um jovem por causa das particularidades locais que influenciam esse risco. Ao se tentar explicar a diferença entre os municípios e estados pela incorporação de suas características, ainda permanece uma variação residual não explicada pelo modelo que é captada em um termo de erro associado à dimensão geográfica 29,30 .

Empregaram-se modelos hierárquicos logísticos dado que a variável resposta se refere à ocorrência ou não de relato de mortalidade jovem em um domicílio i. No primeiro passo, estimou-se um modelo que não considera o ajuste por variáveis independentes e apresenta apenas a variação aleatória no intercepto. Ou seja, o modelo é formalizado por uma equação que contém apenas a variável independente. No segundo e terceiro níveis do modelo, são incluídos os interceptos referentes aos municípios, segundo nível, e estados, terceiro nível.

A especificação do modelo estimado no trabalho segue a apresentação formal apresentada em Raudenbush \& Bryk 30 e apresenta da Equa- ção 2 a Equação 4. A Equação 2 apresenta o modelo geral para o primeiro nível.

$\eta_{i j k}=\beta_{0 \mathrm{jk}+} \beta_{1 \mathrm{jk}} \mathrm{X}_{\mathrm{ijk}}+\mathrm{e}_{\mathrm{ijk}}$

onde: $\eta_{i j k}$ : o log da chance de sucesso (ocorrência do relato de mortalidade jovem pelo domicílio) para o domicílio ${ }_{\mathrm{i}}$, no município $\mathrm{j}$, na Unidade da Federação ${ }_{k}$; $\beta_{0 j}$ : é o intercepto referente ao município ${ }_{\mathrm{j}}$ na Unidade da Federação ${ }_{\mathrm{k}}$; $\beta_{1 \mathrm{j} \mathrm{k}}$ : é o coeficiente, referente ao nível 1 , da direção e associação entre as variáveis; $\mathrm{X}_{\mathrm{ijk}}$ : é o vetor com as características dos domicílios e; $\mathrm{e}_{\mathrm{ijk}}$ : é o efeito aleatório do primeiro nível.

A Equação 3 apresenta a variação observada entre os domicílios dentro de cada um dos municípios analisados.

$\beta_{i j k}=\varpi_{\mathrm{p} 0 \mathrm{k}+} \Sigma \varpi_{\mathrm{pqk}} \mathrm{X}_{\mathrm{qjk}}+\mathrm{r}_{\mathrm{pjk}}$

onde: $\varpi_{\mathrm{p} 0 \mathrm{k}}$ : é o intercepto município na modelagem; $\mathrm{X}_{\mathrm{pqk}}$ : é o vetor com as características dos municípios e; $\mathrm{r}_{\mathrm{ijk}}$ : é o efeito aleatório do segundo nível, que representa a variação nos municípios nos coeficientes estimados do nível ${ }_{1}$.

Neste trabalho, os coeficientes dos níveis $1 \mathrm{e}$ 2 dos modelos hierárquicos podem ser inseridos no nível 3 do modelo de três formas distintas. Optou-se, por assumir que os coeficientes nos níveis 1 e 2 do modelo variam no nível 3 aleatoriamente. Logo, o modelo geral do nível 3, para o nível das Unidades da Federação, é apresentado na Equação 4: 
$\varpi_{\mathrm{pqk}}=\boldsymbol{\Upsilon}_{\mathrm{pq} 0+} \boldsymbol{\Sigma} \boldsymbol{\Upsilon}_{\mathrm{pqs}} \mathrm{X}_{\mathrm{sk}}+\mathrm{u}_{\mathrm{pqk}}$

onde: $\varpi_{\mathrm{p} 0 \mathrm{k}}$ : é o intercepto referente às Unidades da Federação; $\mathrm{X}_{\mathrm{pqk}}$ : é o vetor com as características das Unidades da Federação incluídas no modelo e; $\mathrm{u}_{\mathrm{pqk}}$ : é o efeito aleatório de terceiro nível.

A estratégia de modelagem segue a seguinte ordem. Em primeiro lugar, apresentou-se o modelo que permite decompor a variância entre os três níveis de análise. Ele não inclui variáveis explicativas, e os termos de erro atuam apenas sobre a variável dependente (que descreve o relato de mortalidade jovem pelo domicílio), permitindo fazer a partição da variabilidade total constatada nos dados segundo os níveis de análise (Modelo 1). Com base no modelo nulo, foram desenvolvidos mais quatro modelos, pois há grande heterogeneidade na mortalidade jovem tanto na esfera dos atributos intradomiciliares como espacial e regionalmente. O segundo modelo (Modelo 2) incorpora a variável explicativa no nível intradomiciliar, a saber, o SSED. Assim, o primeiro nível do modelo considera a probabilidade de um domicílio relatar a mortalidade jovem como variável resposta e as características dos domicílios como variáveis explicativas. Assume-se que os coeficientes das equações variam entre os municípios e estados e que tais variações se devem às condições específicas de cada um dos domicílios.

No terceiro modelo (Modelo 3), são incorporadas no nível intradomiciliar as variáveis de controle (sexo e cor/raça do responsável pelo domicílio). Para a variável cor/raça, a categoria de referência é a cor/raça negro (pretos + pardos); para a variável sexo, a categoria de referência é o sexo feminino. O quarto modelo (Modelo 4) inclui as variáveis associadas às características sociodemográficas no nível municipal - o IDH e o tamanho populacional em escala logarítmica. Assume-se que os coeficientes das equações variam entre as unidades de análise e que essas variações são devidas a condições específicas de cada um dos domicílios e dos municípios. Por fim, o quinto modelo (Modelo 5) incorpora as condições de vida na Unidade da Federação, usando o índice de desenvolvimento das Unidades da Federação. Assume-se que os coeficientes das equações variam entre as unidades de análise e que essas variações são devidas às condições específicas de cada domicílio, município e Unidade da Federação.

Com base nas estimativas dos modelos, um segundo passo é calcular probabilidades de ocorrência do evento pelas características observáveis. Nesse sentido, com base nos resultados dos modelos, serão criados tipos ideais de domicílios para fazer comparações entre eles. Para tal, serão utilizados os valores mínimo e máximo das principais variáveis explicativas dos modelos nos níveis domiciliar, municipal e da Unidade da Federação, conjugadas às variáveis de controle no nível dos domicílios.

A medida de ajuste dos modelos utilizada foi o Critério de Informação de Akaike (AIC). O AIC leva em conta a função de máxima verossimilhança do modelo e o número de variáveis explicativas do modelo. O AIC penaliza modelos com muitas variáveis, e modelos com menores valores de AIC são preferíveis. Contudo, é importante ressaltar que não há uma interpretação probabilística que permite dizer que um modelo é significativamente melhor do que outro. O AIC é apenas um mecanismo de comparação de modelos. O AIC é calculado segundo a Equação 5:

$\mathrm{AIC}=-2(\log -$ likelihood $)+2 \mathrm{~K}$

onde: K: é o número de variáveis explicativas.

Para operacionalizar essa proposta analítica, utilizou-se o software HLM 7, disponibilizado pela Scientific Software International (Skokie, Estados Unidos).

\section{Resultados}

\section{Análise descritiva}

A amostra que serve de referência para este estudo contém 6.192.332 domicílios particulares permanentes. Desse total, foram selecionadas 3.422.261 de observações correspondentes aos domicílios que tinham/tiveram na composição da sua estrutura uma pessoa ou mais com idade entre 15 e 29 anos na data do Censo Demográfico de 2010. A Tabela 2 apresenta a distribuição dos domicílios com presença ou não de um jovem e pela ocorrência de óbito nos 12 meses anteriores ao censo. Do total de domicílios expostos ao risco de relatar a mortalidade de um jovem, em apenas $0,26 \%$ deles houve a ocorrência do evento.

A Tabela 3 apresenta as características gerais dos domicílios e de sua composição. Em primei-

Tabela 2

Distribuição dos domicílios da amostra por tipo. Brasil, 2010.

\begin{tabular}{lcc}
\hline Tipo de domicílio & Frequência & $\%$ \\
\hline Domicílio com jovem sem relato de mortalidade jovem & 3.413 .584 & 99,74 \\
Domicílio com jovem e com relato de mortalidade jovem & 5.787 & 0,17 \\
Domicílio sem jovem e com relato de mortalidade jovem & 2.890 & 0,09 \\
Total & 3.422 .261 & 100,00
\end{tabular}


Estatísticas descritivas das características dos domicílios: com e sem mortalidade de jovem. Brasil, 2010.

\begin{tabular}{|c|c|c|c|c|}
\hline & \multicolumn{2}{|c|}{ Domicilio sem mortalidade jovem } & \multicolumn{2}{|c|}{ Domicílio com mortalidade jovem } \\
\hline & Frequência & $\%$ & Frequência & $\%$ \\
\hline \multicolumn{5}{|l|}{ SSED } \\
\hline Baixo & 2.857 .817 & 83,72 & 8.016 & 92,38 \\
\hline Médio & 424.529 & 12,44 & 535 & 6,17 \\
\hline Alto & 131.238 & 3,84 & 126 & 1,45 \\
\hline \multicolumn{5}{|c|}{ Sexo do responsável } \\
\hline Masculino & 2.186003 & 64,04 & 4.302 & 49.58 \\
\hline Feminino & 1.227 .581 & 35,96 & 4.375 & 50.42 \\
\hline \multicolumn{5}{|c|}{ Cor/Raça do responsável } \\
\hline Negro & 1.803 .309 & 52.83 & 5.570 & 64.19 \\
\hline Não negro & 1.610 .275 & 47.17 & 3.107 & 35.81 \\
\hline
\end{tabular}

SSED: Status Socioeconômico do Domicílio.

Fonte: elaboração própria utilizando a amostra do Censo Demográfico 201023

ro lugar, apresenta-se o SSED, separados pela ocorrência ou não do óbito de um jovem. A maior parte da amostra tem SSED baixo (83,72\%). É também nessa proporção dos domicílios que se concentram boa parte das mortes de jovens (92,38\%). Mesmo assim, é possível constatar desigualdades dentre os tipos de domicílios, pois a proporção de domicílios com baixo SSED dentre aqueles com o relato de mortalidade jovem é quase 10 pontos percentuais superior àqueles sem o relato de mortalidade jovem. Para atestar a existência de associação entre essas duas variáveis, antes mesmo do modelo hierárquico, optou-se por fazer um teste qui-quadrado de independência que revelou a existência de relação entre as variáveis.

Em segundo lugar, os resultados indicam que os domicílios que reportaram o óbito de um jovem têm, em sua grande maioria, um responsável do sexo feminino. A variável cor/raça do responsável pelo domicílio também é importante para controlar as diferenças socioeconômicas entre os domicílios. Os resultados mostram diferenças significativas entre os dois tipos de domicílios pela variável cor/raça. Dentre os domicílios com relato de mortalidade jovem, $64 \%$ dos responsáveis são negros, essa proporção dentre os domicílios sem o relato de mortalidade jovem é de $52,83 \%$.

\section{Análise estatística}

Os resultados de todos os modelos são apresentados na Tabela 4. A tabela também apresenta a estimativa do AIC que permite uma comparação entre os modelos.
Os resultados indicam que a variação total observada na probabilidade de relato de mortalidade jovem pelo domicílio foi de 1,0511 (0,9382 do domicílio, 0,0865 do município e 0,0264 da Unidade da Federação). Desse modo, 89,25\% da variação encontrada são em razão das diferenças entre os domicílios, 8,22\% são por conta das diferenças entre os municípios e 2,53\% relacionadas às diferenças entre as Unidades da Federação. Em tal modelo, todos os coeficientes são significativos no nível de $1 \%$. Além disso, no modelo sem a inclusão de variáveis explicativas, foi possível constatar que três em cada grupo de mil domicílios médios da amostra relatam a mortalidade de um jovem.

O segundo modelo incorpora a principal variável explicativa no nível intradomiciliar, ou seja, o SSED. Nota-se que, com a inclusão dessa variável, não há alteração na significância do coeficiente do intercepto. Aqui já é possível estimar a probabilidade de relato de mortalidade jovem pelo domicílio condicionada ao seu SSED. Esssa variável apresenta relação inversa com a probabilidade do relato de mortalidade jovem pelo domicílio, a saber, à medida que se aumenta o valor do SSED, diminui-se a probabilidade do relato de mortalidade jovem pelo domicílio. Verifica-se, para um domicílio com SSED igual a 0 , uma probabilidade de relatar a mortalidade de um jovem de 0,0042. Isso significa dizer que 4,2 domicílios com SSED nulo, em cada grupo de mil, relatariam a mortalidade de um jovem no Brasil no ano anterior à realização do Censo Demográfico de 2010. Ao contrário, quando o SSED do domicílio alcança seu valor máximo, isso é, 1, sua probabilidade de relatar a mortalidade de um 
Resultados dos modelos hierárquicos de três níveis para a probabilidade de relato de mortalidade jovem pelo domicílio. Brasil, 2010.

\begin{tabular}{|c|c|c|c|c|c|}
\hline & Modelo 1 & Modelo 2 & Modelo 3 & Modelo 4 & Modelo 5 \\
\hline \multicolumn{6}{|l|}{ Partição da variância (\%) } \\
\hline Entre Unidades da Federação: u00 & $0,0264(2,53)$ & $0,0165(1,37)$ & $0,0146(1,38)$ & $0,0156(1,49)$ & $0,0136(1,30)$ \\
\hline Entre municípios: rOjk & $0,0865(8,22)$ & $0,1027(9,66)$ & $0,0850(8,07)$ & $0,0664(6,35)$ & $0,0652(6,23)$ \\
\hline Entre domicílios: e0ij & $0,9382(89,25)$ & $0,9437(88,78)$ & $0,9534(90,54)$ & $0,9666(92,51)$ & $0,9672(92,46)$ \\
\hline Variância total & $1,0511(100,00)$ & $1,0629(100,00)$ & $1,0530(100,00)$ & $1,0448(100,00)$ & $1,0460(100,00)$ \\
\hline \multicolumn{6}{|l|}{ Medida de ajuste do modelo } \\
\hline $\mathrm{AIC}$ & 9.199 .078 & 9.406 .132 & 9.449 .532 & 9.326 .660 & 9.376 .694 \\
\hline \multirow[t]{2}{*}{ Intercepto: $\gamma 000$ [OR (IC95\%)] } & 0,002704 & 0,003807 & 0,005731 & 0,001950 & 0,003706 \\
\hline & $(0,003-0,003)$ & $(0,004-0,004)$ & $(0,005-0,006)$ & $(0,001-0,003)$ & $(0,002-0,009)$ \\
\hline \multicolumn{6}{|l|}{ Domicílio [OR (IC95\%)] } \\
\hline \multirow[t]{2}{*}{ SSED } & - & 0,1077 & 0,127342 & 0,113602 & 0,113564 \\
\hline & & $(0,093-0,124)$ & $(0,110-0,147)$ & $(0,099-0,131)$ & $(0,098-0,131)$ \\
\hline \multirow[t]{2}{*}{ Sexo do responsável pelo domicílio } & - & - & 0,565985 & 0,573063 & 0,573216 \\
\hline & & & $(0,544-0,589)$ & $(0,552-0,595)$ & $(0,552-0,596)$ \\
\hline Cor/Raça do responsável pelo & - & - & 0,757594 & 0,755537 & 0,756781 \\
\hline domicílio & & & $(0,725-0,791)$ & $(0,724-0,788)$ & $(0,725-0,790)$ \\
\hline \multicolumn{6}{|l|}{ Município [OR (IC95\%)] } \\
\hline \multirow[t]{2}{*}{ IDH-M } & - & - & - & 2.540552 & 3,143014 \\
\hline & & & & $(1,512-4,270)$ & $(1,746-5,656)$ \\
\hline \multirow[t]{2}{*}{ Log (população residente) } & - & - & - & 1,111367 & 1,099058 \\
\hline & & & & $(1,062-1,163)$ & $(1,048-1,153)$ \\
\hline \multicolumn{6}{|l|}{ Unidade da Federação [OR (IC95\%)] } \\
\hline \multirow[t]{2}{*}{$\mathrm{IDH}$} & - & - & - & - & 0,351142 \\
\hline & & & & & $(0,088-1,401)$ \\
\hline
\end{tabular}

AIC: Critério de Informação de Akaike; IC95\%: intervalo de 95\% de confiança; IDH: Índice de Desenvolvimento Humano; IDH-M: Índice de Desenvolvimento Humano Municipal; OR: odds ratio; PNUD: Programa das Nações Unidas para o Desenvolvimento; SSED: Status Socioeconômico do Domicílio.

Fonte: elaboração própria utilizando a amostra do Censo Demográfico 201023.

jovem cai para 0,0004 . Isso indica que 0,4 domicílio em cada grupo de mil com SSED igual a 1 relataria a mortalidade de um jovem. Assim, entre um domicílio com SSED nulo e outro com SSED máximo há uma diferença de 9,7 vezes na probabilidade de relato de mortalidade de um jovem.

A inclusão das variáveis sexo e raça/cor do responsável pelo domicílio (Modelo 3) permitiu identificar a existência de relação indireta significativa delas com o relato de mortalidade jovem pelo domicílio. Assim, domicílios com mesma condição de SSED, mas cujos responsáveis são do sexo feminino e/ou da cor/raça negra apresentam maiores probabilidades de relatarem a mortalidade jovem.

A inserção das variáveis explicativas no segundo nível (Modelo 4) demonstrou a importância das características dos municípios na variação da probabilidade do relato de mortalidade por um domicílio. Encontrou-se que o IDH-M e o porte populacional do município possuem relação direta com a probabilidade de um domicílio relatar o óbito de um jovem. Logo, domicílios com SSED médio localizados em municípios em melhores condições e cuja população é maior têm maiores probabilidades de relatarem a mortalidade de um jovem.

Por fim, percebe-se que a incorporação da variável IDH da Unidade da Federação (Modelo 5) não altera significativamente a decomposição da variância. Há apenas uma ligeira redução da variabilidade associação às diferenças entre as Unidades da Federação. Ao mesmo tempo, constou-se que o IDH possui relação inversa com a probabilidade do relato de óbito jovem pelo domicílio, ou seja, há maiores chances do relato de mortalidade jovem de domicílios médios em termos de SSED, IDH-M e tamanho populacional para aqueles localizados em Unidades da Federação com menor IDH. O AIC mostra o ajuste dos 
modelos indicando que o Modelo 5, mais completo, é melhor do que o Modelo 4. Entretanto, o modelo com menor AIC é o Modelo 1.

De forma complementar, dimensionou-se como as condições de vida em cada uma desses níveis determinam os diferenciais no risco de morte de um jovem. A Tabela 5 apresenta as probabilidades do relato de mortalidade de um jovem pelo domicílio de acordo com as diferentes condições de vida intradomiciliar, no município e na Unidade da Federação.

Apenas levando em consideração as características dos domicílios, verificou-se que a probabilidade do relato de mortalidade do jovem morador de um domicílio com $\mathrm{SSED}=0$, com responsável da cor/raça negra e do sexo feminino, é 17,92 vezes (A/H na coluna de domicílio) maior do que em um domicílio com SSED = 1, cujo responsável é não negro e do sexo masculino. Para os domicílios cuja única diferença consiste no SSED, a desvantagem para aqueles com SSED = 0 é de 7,7 vezes (A/E na coluna domicílio). Há também uma diferença significativa vinculada apenas ao sexo e à cor/raça dos responsáveis pelos domicílios. Para domicílios com mesmo valor de SSED, se o responsável é mulher e da cor/raça negra, a probabilidade do relato de mortalidade jovem é 32\% (A/B na coluna de domicílio) superior a de um domicílio cujo responsável é uma mulher não negra. De forma similar, dentre os domicílios cujos responsáveis são da cor/raça negra e mulheres, a probabilidade do relato de mortalidade jovem é $76 \%$ (A/C na coluna domicílio) superior àqueles nas mesmas condições, mas em que o responsável é do sexo masculino.

Ao inserir as condições de vida no município, a amplitude das diferenças entre as probabilidades diminuem, mas apresenta aspectos importantes. A probabilidade de um domicílio de tipo A relatar a mortalidade em um município com alto IDH-M é $52 \%$ superior que em um município com baixo IDH-M. Há, também, uma ampliação das diferenças entre domicílios com SSED distintos. Ao se manter constantes as variáveis de controle no âmbito domiciliar, dentre municípios com baixo IDH-M, os domicílios com SSED nulos têm uma probabilidade de relatar a mortalidade jovem 8,86 vezes superior àqueles com SSED igual a 1, proporção que era de 7,88 quando calculadas as probabilidades sem a inserção do IDH-M.

\section{Discussão}

No artigo, discute-se a relação entre condições de vida e mortalidade jovem no Brasil mediante uma abordagem distinta dos estudos existentes sobre o fenômeno no país 1,4,7,31,32,33. Utilizou-se o Censo Demográfico como fonte de dados para mensurar diferenciais de mortalidade jovem sem focar apenas na questão dos homicídios. O Cen-

Tabela 5

Probabilidades de relato de mortalidade jovem pelo domicílio a partir das condições de vida intradomiciliar e das características dos municípios e Unidades da Federação. Brasil, 2010.

\begin{tabular}{|c|c|c|c|c|c|}
\hline \multirow[t]{3}{*}{ Características dos domicílios } & \multirow[t]{3}{*}{ Domicílio } & \multicolumn{2}{|c|}{ Município } & \multicolumn{2}{|c|}{ Unidades da Federação } \\
\hline & & & & Baixo IDH & Alto IDH \\
\hline & & Baixo IDH-M & Alto IDH-M & Baixo IDH-M & Baixo IDH-M \\
\hline$A$ - SSED = 0; negro; feminino & 0,00629 & 0,00346 & 0,00523 & 0,00366 & 0,00457 \\
\hline$B$ - SSED = 0; não negro; feminino & 0,00477 & 0,00262 & 0,00396 & 0,00277 & 0,00346 \\
\hline $\mathrm{C}-\mathrm{SSED}=0$; negro; masculino & 0,00357 & 0,00199 & 0,00300 & 0,00210 & 0,00262 \\
\hline \multirow[t]{2}{*}{ D - SSED = 0; não negro; masculino } & 0,00271 & 0,00150 & 0,00227 & 0,00159 & 0,00199 \\
\hline & & & & Alto IDH-M & Alto IDH-M \\
\hline$E-S S E D=1 ;$ negro; feminino & 0,00081 & 0,00039 & 0,00059 & 0,00069 & 0,00057 \\
\hline$F-\operatorname{SSED}=1$; não negro; feminino & 0,00061 & 0,00030 & 0,00045 & 0,00052 & 0,00043 \\
\hline G - SSED $=1 ;$ negro; masculino & 0,00046 & 0,00022 & 0,00034 & 0,00040 & 0,00033 \\
\hline$H-\operatorname{SSED}=1$; não negro; masculino & 0,00035 & 0,00017 & 0,00026 & 0,00030 & 0,00025 \\
\hline
\end{tabular}

IDH: Índice de Desenvolvimento Humano; IDH-M: Índice de Desenvolvimento Humano Municipal; SSED: Status Socioeconômico do Domicílio.

Fonte: elaboração própria utilizando a amostra do Censo Demográfico 201023 
so Demográfico, ao incluir uma pergunta sobre óbito no domicílio nos últimos 12 meses, possibilitou uma análise que incorporasse as variáveis de domicílio, municípios e estados, o que, ainda, não é possível com as bases de dados administrativas. Todavia, os dados censitários têm duas limitações importantes. Em primeiro lugar, não há informações sobre as condições de vida no período anterior ao óbito e, caso ele tenha causado um impacto negativo nas condições de vida do domicílio, pode-se estar observando uma relação espúria. Em segundo lugar, temos as informações sobre o domicílio e o município de residência, mas como há grande heterogeneidade nas condições de vida municipais é possível que exista algum problema de confundimento por conta da incapacidade de se controlar pelas condições contextuais e ambientais dos domicílios dentro de cada um dos municípios e estados. De todo modo, os resultados indicam a importância de considerar não apenas as condições de vida dos municípios e estados, mas também a situação familiar e domiciliar relacionadas a maiores probabilidades de óbitos de jovens adultos no Brasil.

A proposta metodológica permitiu identificar o grau de importância de diferentes esferas das condições de vida dos jovens na definição dos diferenciais regionais de mortalidade e avaliar a importância de fatores socioeconômicos domiciliares na definição de diferenciais de mortalidade nesse grupo etário. Os resultados mostram que domicílios de nível socioeconômico mais baixo, localizados em municípios mais ricos, têm maiores chances de observar um óbito de um jovem adulto no ano anterior à realização do Censo Demográfico. Não apenas o indicador de nível socioeconômico é importante para explicar o diferencial de mortalidade, mas outras características relacionadas à maior vulnerabilidade - como responsável do sexo feminino e de raça/ cor não branca tem forte relação com maiores probabilidades de óbito de jovens no domicílio, algo que merece maior investigação para tentar compreender como tais características específicas afetam o evento mortalidade jovem. Portanto, cabe discutir se os domicílios chefiados por mulheres e ou por pessoas de cor/raça negra possuem outras características que esta proposta metodológica não mensurou e que afetaram de forma conjugada a mortalidade jovem, por exemplo, se há a presença.

Estudo sobre outro país aponta que a fase de transição para a vida adulta é marcada tanto por maiores riscos de lesões como de mortalidade para a população jovem e que esses riscos estão associados diretamente a questões de background familiar bem como vinculados a características dos arranjos domiciliares nos quais os jovens adultos estão inseridos, como a idade precoce da saída da casa dos pais e como a existência de apenas um dos cônjuges no domicílio 34.

A estratégia de pesquisa permitiu verificar e quantificar o papel das características dos domicílios, municípios e dos estados na variabilidade das chances de um óbito de jovens no Brasil e identificar quais atributos contribuem para a determinação desses diferenciais. Os resultados apontam que as diferenças nos níveis de status socioeconômico entre os domicílios são responsáveis por mais de $90 \%$ da variabilidade no risco de relato de óbito jovem pelo domicílio. Isso significa que a quase totalidade do diferencial de mortalidade encontrada é explicada pelos diferenciais de vida nos domicílios. Nessa mesma direção, os resultados indicam que domicílios com condições de vida muito baixas, menor SSED, têm 18 vezes mais chances de relatar a mortalidade jovem do que àqueles com nível socioeconômico alto.

De forma complementar, as condições de vida nos municípios conjugadas ao seu porte populacional afetam de $6 \%$ a $8 \%$ dos diferenciais de mortalidade. Nessa esfera, concluiu-se que domicílios localizados em municípios com melhores condições de vida apresentam maiores riscos de relatar a mortalidade jovem, independentemente do seu nível socioeconômico domiciliar e das características dos responsáveis pelo domicílio. Além disso, constatou-se que, nas cidades de médio e grande porte, a desvantagem dos domicílios em piores condições socioeconômicas é ligeiramente superior, aspecto que corrobora com a perspectiva empírica apresentada por estudo que realizou uma análise ecológica dos homicídios de jovens e adultos entre 1999 e 2010, nos municípios brasileiros, e encontrou maiores taxas dentre os municípios de maior porte populacional 31,32,33,35,36,37,38. Contrariamente ao que ocorre na esfera domiciliar, o IDH-M demonstrou ter relação positiva com o risco de relato de óbito jovem pelo domicílio. Tem-se como hipótese para essa relação aparentemente não esperada que a mortalidade jovem é afetada de maneira significativa pelos eventos associados à violência e, sobretudo a violência associada ao tráfico de drogas, que concentra uma parcela significativa das mortes por causas violentas 7,15,38. Tal evento, a despeito do processo de interiorização e disseminação apontados por estudo recente, ocorre de modo mais sistemático nos grandes centros urbanos 1. Contudo, internamente às cidades, no caso dos homicídios, há uma concentração das mortes por homicídio em localidades que concentram também domicílios mais pobres, o que justificaria a relação negativa entre o evento 
mortalidade jovem e as condições de vida intradomiciliar e, por outro lado, a relação positiva com o IDH-M.

Por fim, as condições de vida nas Unidades da Federação afetam cerca de $1 \%$ dos diferenciais de mortalidade jovem. Ao serem incluídas as condições de vida nos estados (Unidades da Federação), as disparidades na probabilidade do relato de mortalidade jovem pelos domicílios diminuem. Da mesma forma, persiste a manutenção da relação entre condições de vida e seu impacto nas chances de se relatar o óbito de um jovem pelo domicílio nas Unidades da Federação. Entretanto, para domicílios com condições de vida baixas tanto no nível domiciliar (baixo SSED) quanto no município (baixo IDH-M), a probabilidade do relato de mortalidade jovem, se ele estiver localizado em uma Unidades da Federação com alto IDH, é $24 \%$ superior a daqueles localizados em Unidades da Federação com baixo IDH. Porém, para domicílios com condições de vida melhores no nível intradomiciliar e municipal é melhor estar localizado em Unidades da Federação com condições de vida melhores. Embora este estudo tenha apontado o menor poder explicativo das condições de vida nos estados na definição do risco de morte de jovens no Brasil, estudo focado na relação entre desigualdade de renda e risco de morte entre jovens e adultos nos Estados Unidos aponta para a relação direta em gasto com bem-estar e educação e o risco de morte entre jovens e adultos nos estados americanos 39 .

Os resultados deste estudo completam as evidências apresentadas na literatura ao descrever tanto a existência de altas taxas de mortalidade jovem no território brasileiro quanto evidências de diferenciais no risco de morte com base nas características dos domicílios e por causa das diferenças entre as condições de vida nos municípios e nas Unidades da Federação 1,4,7,8,9,10,11,31,34,35,36,40. Trabalhos que focam no nível individual têm identificado também a existência de diferenciais de mortalidade dentro do grupo etário jovem em razão de atributos pessoais como sexo e cor/raça e comportamentais como uso de álcool e drogas 37,38. Desse modo, ao concluir que jovens pobres moradores de áreas ricas têm mais risco de mortalidade, este estudo aponta para a necessidade de se aprimorar o conhecimento acerca dos efeitos da desigualdade na mortalidade em tal grupo etário. Medidas e metodologias que captem as diferenças de oportunidades e de acesso podem contribuir para uma compreensão mais profunda dos efeitos e da seletividade da mortalidade no grupo etário jovem no país.

As taxas de mortalidade de jovens adultos mais altas são encontradas em todas as unidades da federação, tanto na parte litorânea quanto na parte interiorana do país 30,36. Diferentemente dos processos de concentração dos homicídios nas grandes cidades e centros urbanos da região Sudeste, que caracterizou a violência no país nas décadas de 1980 e 1990, a partir de 2003, ocorrem os processos de disseminação, com a incidência de altas taxas de homicídios em municípios de pequeno e médio porte, representando a interiorização do fenômeno, por sua incidência não somente nas metrópoles e suas regiões metropolitanas, mas também em cidades do interior dos estados. A reorganização da violência descreve um processo complexo que extrapola esses fenômenos, ou seja, a reorganização verificada nos anos 2000 sugere também lógicas de aglomeração com a presença de efeitos de contágio e estruturação de clusters de homicídios em áreas que recentemente apresentaram avanço econômico e/ou reorganização espacial 38,40,41.

\section{Agradecimentos}

Ao CNPq e Fapemig pelo financimento e aos pareceristas anônimos pelos comentários e sugestões. 


\section{Referências}

1. Waiselfisz JJ. Mapa da violência 2013: homicídios e juventude no Brasil. Rio de Janeiro: Centro de Estudos Latino-Americanos; 2013.

2. Campos MR, von Doellinger VR, Mendes LVP, Costa MFS, Pimentel TG, Schramm JMA. Morbidity and mortality associated with injuries: results of the Global Burden of Disease study in Brazil, 2008. Cad Saúde Pública 2015; 31:121-36.

3. Chiavegatto Filho ADP, Beltrán-Sánchez H, Kawachi I. Racial disparities in life expectancy in Brazil: challenges from a multiracial society. Am J Public Health 2014; 104:2156-62.

4. Souza ERD, Gomes R, Silva JG, Correia BSC, Silva MMAD. Morbidity and mortality of young Brazilian men due to aggression: expression of gender differentials. Ciênc Saúde Coletiva 2012; 17:3243-8.

5. Krug EG, Dahlberg LL, Mercy JA, Zwi AB, Lozano R. World report on violence and health. Geneva: World Health Organization; 2002.

6. Cerqueira D, Soares R. The welfare cost of homicides in Brazil: accounting for heterogeneity in the willingness to pay for mortality reductions. Health Econ 2016; 25:259-76.

7. Minayo MCS. Seis características das mortes violentas no Brasil. Rev Bras Estud Popul 2009; 26:135-40.

8. Adorno S. A criminalidade urbana violenta no Brasil: um recorte temático. Revista Brasileira de Informação Bibliográfica em Ciências Sociais - BIB 1993; 35:299-342.

9. Zaluar A. Um debate disperso: violência e crime no Brasil da redemocratização. São Paulo Perspect 1999; 13:3-17.

10. Cruz MVG, Batitucci EC. Homicídios no Brasil. Rio de Janeiro: Editora FGV; 2007.

11. Cano I, Santos N. Violência letal, renda e desigualdade social no Brasil. Rio de Janeiro: 7 Letras; 2001.

12. Soares GAD. Não matarás: desenvolvimento, desigualdade e homicídios. Rio de Janeiro: Editora FGV; 2008.

13. Souza ER, Minayo MCS. Morbimortalidade de jovens de 15 a 29 anos por violências e acidentes no Brasil: situação atual. Tendências e perspectivas. Brasília: Organização Pan-Americana da Saúde/ Centro Latino-Americano de Estudos de Violência e Saúde Jorge Careli, Escola Nacional de Saúde Pública Sergio Arouca, Fundação Oswaldo Cruz; 2007.

14. Barata RBR, Ribeiro MCSA, Moraes JC. Desigualdades sociais e homicídios em adolescentes e adultos jovens na cidade de São Paulo em 1995. Rev Bras Epidemiol 1999; 2:50-9.

15. Beato C, Reis I. Desigualdade, desenvolvimento socioeconômico e crime. In: Enriques R, organizador. Desigualdade e pobreza no Brasil. Rio de Janeiro: Instituto de Pesquisa Econômica Aplicada; 2000. p. 385-403.

16. Duarte EC, Tauil PL, Duarte E, Souza MC, Monteiro RA. Mortalidade por acidentes de transporte terrestre e homicídios em jovens das capitais das regiões Norte e Centro-oeste do Brasil, 1980-2005. Epidemiol Serv Saúde 2008; 17:7-20.
17. Wilmoth JR, Dennis M. Social differences in older adult mortality in the United States: questions, data, methods, and results. In: Robine JM, Crimmins EM, Horiuchi S, Zeng YI, editors. Human longevity, individual life duration, and the growth of the oldest-old population. Dordrecht: Springer; 2006. p. 297-332.

18. Preston S, Taubman P. Socioeconomic differences in adult mortality and health status. In: Preston S, Martin L, editors. The demography of aging. Washington DC: National Academy Press; 1994. p. 279318.

19. Wood CH, Carvalho JAM. Desigualdade de renda e expectativa de vida. In: Wood CH, Carvalho JAM, organizadores. A demografia da desigualdade no Brasil. Rio de Janeiro: Instituto de Pesquisa Econômica Aplicada; 1994. p. 101-19.

20. Sastry N. Trends in socioeconomic inequalities in mortality in developing countries: the case of child survival in São Paulo, Brazil. Demography 2004; 41:443-64.

21. Krieger N, Williams DR, Moss NE. Measuring social class in US publichealth research: concepts, methodologies, and guidelines. Ann Rev Public Health 1997; 18:341-7.

22. Vergara M, Benach J, Muntaner C. Desigualdad en salud: la mayor epidemia del siglo XXI. Papeles 2008; 103:29-40.

23. Instituto Brasileiro de Geografia e Estatística. Censo Demográfico 2010: características de população e dos domicílios. Resultados do universo, 2011. http://www.ibge.gov.br/home/estatistica/popu lacao/Censo2010/caracteristicas_da_populacao/ resultados_do_universo.pdf (acessado em 10/Nov/ 2013).

24. Queiroz BL, Sawyer DO. O que os dados de mortalidade do Censo de 2010 podem nos dizer? Rev Bras Estud Popul 2012; 29:225-38.

25. Marmot M. Social determinants of health inequalities. Lancet 2005; 365:1099-104.

26. Blane D. An assessment of the Black Report's explanations of health inequalities. Sociol Health Illn 1985; 7:423-45.

27. Lauderdale DS. Education and survival: birth cohort, period and age effects. Demography 2001; 38:551-61

28. Christenson BA, Johnson NE. Educational inequality in adult mortality: an assessment with death certificate. Demography 1995; 32:215-29.

29. Diez-Roux AV. Multilevel analysis in public health research. Ann Rev Public Health 2000; 21:171-92.

30. Raudenbush S, Bryk A. Hierarchical linear models: applications and data analysis methods. Sage: Thousand Oaks; 2002.

31. Duarte EC, Garcia LP, Freitas LRS, Mansano NH, Monteiro RA, Ramalho WM. Associação ecológica entre características dos municípios e o risco de homicídios em homens adultos de 20-39 anos de idade no Brasil, 1999-2010. Ciênc Saúde Coletiva 2012; 17:2259-68

32. Andrade LT, Diniz AMA. A reorganização espacial dos homicídios no Brasil e a tese da interiorização. Rev Bras Estud Popul 2013; 30 Suppl:S171-91. 
33. Mansano NH, Gutierrez MMU, Ramalho W, Duarte EC. Homicídios em homens jovens de 10 a 24 anos e condições sociais em municípios do Paraná e Santa Catarina, Brasil, 2001-2010. Epidemiol Serv Saúde 2013; 22:203-14.

34. Remmes H, Martikainem P. Young adult's own and parental social characteristics predict injury morbidity: a register-based follow-up of 135000 men and women. BMC Public Health 2015; 15:429.

35. Moura EC, Gomes R, Falcão MTC, Schwarz E, Neves ACM, Santos W. Desigualdades de gênero na mortalidade por causas externas no Brasil, 2010. Ciênc Saúde Coletiva 2015; 20:779-88.

36. Soares Filho AM. Vitimização por homicídios segundo características de raça no Brasil. Rev Saúde Publica 2012; 45:745-55.

37. Abreu DMX, César CC, França EB. Diferenciais entre homens e mulheres na mortalidade evitável no Brasil (1983-2005). Cad Saúde Pública 2009; 25:2672-82.

\begin{abstract}
This study analyzed the relationship between household socioeconomic status and local social conditions and risk of death in Brazilian youth 15 to 29 years of age. Mortality data from the previous 12 months collected by the 2010 Population Census were used for the analysis at the local level. Data analysis was based on hierarchical models that allowed identifying the role of household social conditions and municipality and state of residence on mortality in young Brazilians. Differences in socioeconomic status between households accounted for more than $90 \%$ of the variability in risk of death. Residing in socioeconomically deprived areas also increased the odds of death among young people from households of any socioeconomic status.
\end{abstract}

Young Adult; Social Conditions; Mortality
38. Murray J, Cerqueira DRC, Kahn T. Crime and violence in Brazil: systematic review of time trends, prevalence rates and risk factors. Aggress Violent Behav 2013; 18:471-83.

39. Kim D. The associations between US state and local social spending, income inequality, and individual all-cause and cause-specific mortality: the National Longitudinal Mortality Study. Prev Med 2016; 84:62-8.

40. Reichenheim ME, Souza ER, Moraes CL, Mello Jorge MHP, Silva CMFP, Minayo MCS. Violence and injuries in Brazil: the effect, progress made, and challenges ahead. Lancet 2011; 377:1962-75.

41. Carvalho AXY, Silva GDM, Almeida Júnior GR, Albuquerque PHM. Taxas bayesianas para o mapeamento de homicídios nos municípios brasileiros. Cad Saúde Pública 2012; 28:1249-62.

\section{Resumen}

En este artículo, se analizó la relación entre el nivel socioeconómico de los domicilios y las condiciones sociales locales, con el riesgo de muerte de jóvenes brasileños. Se utilizaron los datos de fallecimientos ocurridos en los últimos 12 meses, recogidos por el Censo Demográfico de 2010, para realizar el análisis a nivel local. El análisis de los datos se realizó en base a modelos jerárquicos que posibilitaron identificar la contribución de las condiciones sociales de los domicilios, municipios y estado de residencia en la mortalidad de jóvenes en Brasil. Los resultados indican que las diferencias en los niveles de status socioeconómico entre los domicilios son responsables de más de un $90 \%$ de la variabilidad, en el riesgo de un informe de fallecimiento de un joven por el domicilio. Asimismo, los resultados muestran que residir en una localidad con bajo nivel socioeconómico aumenta las posibilidades de ocurrencia de un informe de fallecimiento de jóvenes por los domicilios de cualquier nivel social durante el período.

Adulto Joven; Condiciones Sociales; Mortalidad
Recebido em 07/Jul/2015

Versão final reapresentada em 14/Jan/2016

Aprovado em 02/Mar/2016 